\title{
Determination of the Influence of Chronic Periodontitis on Pulp Sensibility by Means of Electric and Thermal Cold Testing
}

\author{
Vanessa C.Q. Neves, DDS, MS, ${ }^{*}$ Benedicto E.C. Toledo, DDS, MS, PbD, ${ }^{\dagger}$ \\ Gabriela A.C.G. Camargo, DDS, MS, PhD, ${ }^{\neq}$Alessandra A. Souza, DDS, MS, PbD, ${ }^{\neq}$ \\ and Elizangela P. Zuza, DDS, PhD
}

\begin{abstract}
Ahstract
Introduction: Communication between pulp and periodontal tissue has been well established. However, it is unknown when periodontal disease begins to affect the clinical response of pulp tissue. The aim of this study was to assess the influence of periodontal severity on pulp sensibility by means of electric and thermal cold testing. Methods: The teeth assessed in this study were allocated into 3 groups considering radiographic alveolar bone loss $(\mathrm{ABL})$ as follows: slight periodontitis $(\mathrm{SP}, \mathrm{ABL} \leq 7 \mathrm{~mm}$ without reaching the apex, $n=25$ ), moderate periodontitis ( $\mathrm{BBL}>7 \mathrm{~mm}$ without reaching the apex, $n=23$ ), and severe periodontitis (SvP, ABL $>7$ reaching the apex, $n=8)$. Gingival recession (GR), probing depth (PD), and clinical attachment level (CAL) were also measured. Results: The results showed higher levels of PD and CAL in the SvP group compared with the SP group $(P<.05)$, with no significant difference in GR $(P>.05)$. The SvP group showed significant ABL compared with the other groups $(P>.05)$. The SP group showed a significant number of teeth with a positive pulp response, whereas the SvP group showed a significant number of teeth with a negative pulp response $(P<.05)$; no significant differences were observed between the thermal cold and electric tests $(P>.05)$. Conclusions: Within the limits of this study, it can be concluded that pulp clinical involvement with a negative response to thermal cold and electric testing occurs only in the most advanced stage of chronic periodontitis with apical involvement. (J Endod 2017;43:1802-1805)
\end{abstract}

\section{Key Words}

Chronic periodontitis, dental pulp, endodontics
The he communication between pulp and periodontal tissues is well established in the literature. The effects of pulp alterations on the periodontium are well-documented

$(1,2)$, whereas the influence of periodontal disease on pulp tissue needs further assessment, with new forms of clinical and histologic investigations being required. Injuries to the pulp tissues caused by lasting and continuous stimuli or chemical irritants that act directly on the dentin can alter pulp physiology (3). Periodontitis can be considered a chronic and continuous stimulus on the pulp (4). Some authors have assessed the pulp histopathologic condition in teeth with different severities of chronic periodontitis. It was reported that the gradual progression of the disease causes pulp alterations, such as reaction fibrosis, dystrophic mineralization, atrophy, and mononuclear inflammatory infiltration (4).

When the pulp is in the degenerative phase, it is unlikely that its condition improves, signaling an irreversible abnormality. In this context, the response of pulp can be influenced by electric and thermal stimuli. Therefore, the determination of vitality status and pulp health is the most commonly indicated approach for a differential diagnosis (5). The thermal cold vitality test can be used to reproduce the symptoms reported by patients when testing pulp sensibility (6). In this way, the diagnosis can be reliably made with limited false-negative results; specifically, a negative reaction represents necrotic pulp in $90 \%$ of cases $(5,7,8)$.

The electric test provides microscale quantitative data by measuring the neural response to stimuli. Therefore, this test can be used as an auxiliary means to diagnose pulp health or necrosis. Negative responses are highly likely linked to necrotic or severely diseased pulp and represent irreversible stages of degeneration (9). Thus, it is important that clinicians understand and know how to interpret the electric test, which is performed using an electric charge, because false-negative or positiveresponses might occur (10).

Because of the scarcity of studies that assess the influence of periodontal disease on the pulp, the assessment of this condition is believed to be important, not only when the destruction reaches the dental apex but also for different levels of attachment loss in chronic periodontitis (4). The optimal understanding of this interrelation is crucial and

From the *Department of Dentistry, Educational Foundation of Barretos (UNIFEB), School of Dentistry, Barretos, São Paulo, Brazil; ${ }^{\dagger}$ Department of Diagnosis and Surgery, São Paulo State University (UNESP), School of Dentistry, Araraquara, Brazil; and ${ }^{\ddagger}$ Department of Periodontology, Fluminense Federal University, School of Dentistry, Nova Friburgo, Rio de Janeiro, Brazil.

Address requests for reprints to Dr Elizangela P. Zuza, Department of Periodontology, Fluminense Federal University (UFF), Nova Friburgo, Rio de Janeiro, Brazil. E-mail address: elizangelazuza@yahoo.com.br

0099-2399/\$ - see front matter

Copyright (C) 2017 American Association of Endodontists.

http://dx.doi.org/10.1016/j.joen.2017.07.006 
may make it applicable in daily clinical practice, verifying the hypothesis that it is not necessary for periodontitis to reach the dental apex to cause pulp alterations (5).

Pulp sensibility testing is the most indicated approach for a differential diagnosis of the relationship between the pulp and the periodontium. However, according to some authors (5), a single test is generally insufficient to make a conclusive diagnosis; rather, 2 or more tests should be used to confirm the true condition of the dental pulp. The aim of this study was to assess the influence of chronic periodontitis on the pulp response by means of electric and cold thermal testing.

\section{Material and Methods}

This study was approved by the UNIFEB Research Ethics Committee (protocol no. 883.469/2014). This work was performed in accordance with the Code of Ethics of the World Medical Association (Declaration of Helsinki) for experiments involving humans. Informed consent was obtained for experimentation, and the privacy rights of human subjects were preserved. Fifty-six single-rooted teeth were assessed. The teeth were selected from 11 patients with chronic periodontitis (11) who sought treatment at either the dentistry course clinics at UNIFEB or private practices. Five patients were men, and 6 were women, with ages ranging between 31 and 66 years.

To be included in the study, teeth had to be single rooted. Patients with pacemakers and cardiac monitoring devices as well as those who had received periodontal treatment in the previous 6 months were excluded. Multirooted and carious teeth or those with restorations were also excluded. The selection criteria did not necessarily exclude all possible reasons, such as accidental trauma or cracks. An examiner (E.P.Z.) was trained to measure the periodontal parameters, and another examiner (V.C.Q.N.) was trained to assess pulp sensibility using electric and cold thermal testing. The criteria were explained, and training was performed, with an expert examiner being considered the "gold standard."

\section{Assessment of Periodontal Parameters}

The periodontal condition determination was based on the following clinical parameters (12): gingival recession (GR, the distance between the cementoenamel junction and the gingival margin, measured in millimeters), probing depth (PD, the distance from the edge of the gingival margin to the bottom of the gingival sulcus, measured in millimeters), bleeding on probing (BOP, present or absent until 30 seconds after probing), and clinical attachment level (CAL, the distance from the cementoenamel junction to the bottom of the pocket measured in millimeters, corresponding to the sum of the GR and PD). A periodontal probe (PCPUNC15BR; Hu-Friedy, Chicago, IL) was used to measure the PD, BOP, GR, and CAL in the following 6 sites for each tooth: mesiobuccal, medial, mesiolingual, distolingual, lingual, and distal vestibular.

Alveolar bone loss (ABL) assessment was performed by means of periapical X-rays using the paralleling technique. ABL was considered when the distance between the cementoenamel junction and the alveolar bone crest was $>2 \mathrm{~mm}$ (13). The ABL extension was measured in the proximal surfaces (medial and distal) from the cementoenamel junction to the most apical point of the bone crest using a caliper rule (Mitutoyo, Tokyo, Japan) and an adequate negatoscope. Only the largest ABL measure was recorded on the chart. The radiographic ABL extension was classified as follows: slight periodontitis (SP, $\mathrm{ABL} \leq 7 \mathrm{~mm}$ without reaching the apex), moderate periodontitis (MP, ABL $>7 \mathrm{~mm}$ without reaching the apex), and severe periodontitis (SvP, ABL $>7 \mathrm{~mm}$ reaching the apex). The groups of teeth were separated according to the ABL level based on the study by Zuza et al (4) (Table 1).
TABLE 1. Study Groups and Alveolar Bone Loss (ABL) Criteria

\begin{tabular}{cc}
\hline Groups & Criteria \\
\hline $\begin{array}{c}\text { Slight periodontitis } \\
(n=25)\end{array}$ & $\mathrm{ABL} \leq 7 \mathrm{~mm}$, without reaching the \\
apex \\
$\begin{array}{c}\text { Moderate periodontitis } \\
(n=23)\end{array}$ & $\mathrm{ABL}>7 \mathrm{~mm}$, without reaching the \\
apex \\
$\begin{array}{c}\text { Severe periodontitis } \\
(n=8)\end{array}$ & $\mathrm{ABL}>7 \mathrm{~mm}$, reaching the apex \\
\hline
\end{tabular}

\section{Pulp Sensibility Assessment: Electric and Cold Thermal Testing}

To assess pulp sensibility and thereby obtain a more conclusive diagnosis, pulp status determination was performed using both electric and cold thermal testing. The materials used for the tests were the Micro-Controlled Pulp Tester Digital TP-10 (Microeletrônica Ind. e Com. Ltda, Belo Horizonte, Brazil) and Endo-Frost cold spray (Roeko, Langenau, Germany).

To perform the thermal cold test, a cooling spray gas was used. After isolating the tooth crown with cotton rolls and drying its surface, clinical tweezers and a small cotton ball saturated by the cooling gas were applied to the central point of the cervical third of the anatomic crown $(9,14)$. The cotton ball was pressed on the tooth for approximately 3 seconds, and the stimulus position was maintained to determine either the immediate tooth response or the response up to 10 seconds later.

The electric test was conducted using an electric charge by means of a specific device that directly stimulates sensory fibers. This test has been shown to be effective in diagnosing pulp sensibility, and the stimuli are gradually microcontrolled, which alleviates the painful effects according to the manufacturer. There is a digital counter and an auditory beeper to indicate the level of stimuli. This device was used in the normal mode. Thus, the electric charge reading was performed using the device screen, considering the manufacturer's instructions for single-rooted teeth, and the tooth was considered vital if the charge was higher than 30 . These parameters were carefully followed to prevent the electric charge from reaching the periodontal fibers, which could influence the research results (15).

The electric test was performed at the central point of the cervical third of the anatomic crown (14) using toothpaste as an electric conductor on the dental surface. The patients were instructed to release the handle of the test device as soon as they felt the electric stimulus, at which point the charge was registered.

To avoid individual sensitivity variation, thermal cold and electric tests were repeated on contralateral healthy teeth. There was a 5 -minute interval between the tests performed in the same tooth. All the data were recorded on a form specially designed for the study.

\section{Statistical Analysis}

Continuous PD, GR, BOP, and CAL data were statistically analyzed using analysis of variance ( 1 criterion) followed by the Tukey test. The frequency of the pulp response to cold and electric stimuli and the comparison between the tests were calculated using the chi-square test. BioEstat 5.0 software (Mamirauá, Tefé, AM, Brazil) was used, and the significance level was set at $5 \%$.

\section{Results}

Fifty-six teeth that met the inclusion criteria were assessed in the present study. The results showed that the MP and SvP groups presented similar PD and CAL values, whereas the SvP group presented significantly higher ABL values in comparison with the other groups 
$(P<.05)$. There were no significant differences in GR among the 3 groups $(P>.05)$ (Table 2$)$.

Both thermal cold and electric tests showed a higher number of teeth with a negative pulp response for the SvP group and a significantly higher number of teeth with a positive pulp response in the SP group $(P<.05$, Table 3$)$. The cold thermal and electric tests showed no statistically significant differences with respect to positive and negative responses to the tests $(P>.05$, Table 4$)$.

\section{Discussion}

The results presented in the study show a clinical correlation between pulp response and the worsening of periodontal disease and are consistent with previous studies $(4,16,17)$. Moreover, histologic studies have confirmed the existence of pulp alterations in the presence of periodontal disease (4, 16-18). Despite these findings, there is no consensus in the literature regarding how much one influences the other (19). Certain authors have stated that periodontal disease has no influence on the pulp; however, pulp degeneration could occur because of systemic factors (19).

The findings in this study show that pulp remains vital until the most severe stage of periodontitis. In cases of SP and SvP (with no apical involvement), there was no prevalence of a negative pulp response, whereas in MP (with apical involvement), significant negative pulp responses were observed for both the cold thermal and electric tests. Our clinical results corroborate those from Langeland et al (18). These authors, despite observing pathological alterations in the pulp tissue, concluded that it did not succumb so long as circulation via the main canal was unaffected. These findings were also confirmed in the clinical part of the same study (18) in which the authors reported that a more severe progression of periodontitis was significantly related to a negative pulp response.

Jansson et al $(20,21)$ stated that single-rooted teeth with periapical radiolucent lesions were significantly correlated to deep periodontal pockets and worse radiographic bone loss, a result that was also confirmed in the present study. Our results suggest that pulp condition depends on the severity of active periodontal disease and progressive bone reabsorption.

In a second study performed by Zuza et al (4), which used the same classification scheme used for this study, the gradual progression of chronic periodontitis led to histopathological modifications in the pulp root in the most advanced cases of bone loss with apical involvement. However, these authors showed that the coronal pulp remained within the normal range in all stages of periodontitis (ie, SP, MP, and SP), demonstrating a significant defense capability of pulp tissue.

Other authors (19) also stated that pulp tissue usually reacts to pulp calcification formations and pulp chamber and root canal atresia. The periodontium was reported to be the most vulnerable to pulp

TABLE 2. Periodontal Parameters

\begin{tabular}{lrrr}
\hline & \multicolumn{3}{c}{ Groups } \\
\cline { 2 - 4 } Parameters $(\mathrm{mm})$ & SP & MP & \multicolumn{1}{c}{ SvP } \\
\hline Gingival recession & $3.16 \pm 1.57^{\mathrm{a}}$ & $4.3 \pm 2.51^{\mathrm{a}}$ & $5.87 \pm 1.95^{\mathrm{a}}$ \\
Probing depth & $3.72 \pm 1.27^{\mathrm{a}}$ & $5.82 \pm 2.20^{\mathrm{b}}$ & $6.25 \pm 3.49^{\mathrm{b}}$ \\
Clinical attachment & $7.2 \pm 1.84^{\mathrm{a}}$ & $10.08 \pm 2.17^{\mathrm{b}}$ & $12.0 \pm 2.97^{\mathrm{b}}$ \\
$\quad$ level & & & \\
Alveolar bone loss & $5.24 \pm 1.45^{\mathrm{a}}$ & $9.21 \pm 0.95^{\mathrm{b}}$ & $11.37 \pm 1.76^{\mathrm{c}}$ \\
\hline
\end{tabular}

MP, moderate periodontitis; SP, slight periodontitis; SvP, severe periodontitis

Values shown are mean \pm standard deviation. Analysis of variance (1 criterion) followed by the Tukey test was performed. Different superscript letters indicate statistically significant differences among groups $(P<.05)$
TABLE 3. Pulp Responses in the Different Study Groups Submitted to Electric and Thermal Cold Stimuli

\begin{tabular}{lcc}
\hline \multicolumn{1}{c}{ Groups } & No. of teeth (PV) & No. of teeth (NV) \\
\hline Thermal cold test & $25^{*}$ & 0 \\
SP & 20 & 3 \\
MP & 2 & $6^{*}$ \\
SvP & $23^{\dagger}$ & \\
Electric test & 16 & 2 \\
SP & 2 & 7 \\
MP & 2 & $6^{\dagger}$ \\
SvP &
\end{tabular}

MP, moderate periodontitits; NV, negative vitality; PV, positive vitality; SP, slight periodontitis; SvP, severe periodontitis

*Statistically significant difference $\left(\chi^{2}=25.5393\right.$; Degree of freedom $=2 ; P<.0001 ; \chi^{2}$ adjusted residual, $\alpha=0.01$ ).

${ }^{\dagger}$ Statistically significant difference $\left(\chi^{2}=14.1379\right.$; Degree of freedom $=2 ; P=.0009 ; \chi^{2}$ adjusted residual, $\alpha=0.01$ ).

necrosis products, tending to suffer reabsorption. Because of the pulp defense reaction to contamination caused by periodontal pathogens, pulp calcifications or pulp chamber and/or root canal atresia result in the production of reactive dentin. This effect may suggest a cause-effect relationship between periodontal disease and pulp necrosis.

Teeth affected by occlusal trauma and those that were restored but still showed signs of decay or prosthetic crowns were not considered in the determination of the influence of chronic periodontal disease on the pulp. The chronic periodontal disease patients in the present study had a median age of 60.5 years (11), and it is known that the severity of the condition increases with age. Therefore, it can be concluded that the pulp of the selected teeth had already begun to suffer age-related alterations (22). False-positive pulp sensibility results are frequently encountered in multirooted teeth, so these teeth were excluded $(5,9)$. The selection criteria did not necessarily exclude all possible reasons for pulp necrosis, such as accidental trauma or cracks. This creates the possibility that there may be a tooth that responds as nonvital yet may still fit the selection criteria.

To assess pulp sensibility, a thermal cold stimulus with a cooling spray was used. According to some authors $(5,7,8)$, the spray allows a reliable diagnosis with few false-negative results; true-negative results reliably predict necrotic pulp in $90 \%$ of cases. To confirm the pulp condition, an electric test using an electric charge with a direct stimulus on sensory fibers was used. According to Weisleder et al (8), the electric test (Micro-Controlled Pulp Tester Digital TP-10) and the thermal cold stimuli (Endo-Frost) are equally effective in confirming a diagnosis of the condition of the pulp, a result that was confirmed in the present study. The electric test aims to stimulate a response from the afferent fibers inside the pulp, indicating only whether they are vital. This assessment provides no information about vascular supply, which is the real

TABLE 4. Comparison between Cold Thermal and Electric Tests

\begin{tabular}{lcccc}
\hline Groups & Pulp vitality & Cold test, $\boldsymbol{n}$ & Electric test, $\boldsymbol{n}$ & $\boldsymbol{P}$ value \\
\hline SP & Positive & 25 & 23 & $>.05$ \\
& Negative & 0 & 2 & \\
MP & Positive & 20 & 16 & $>.05$ \\
& Negative & 3 & 7 & \\
SvP & Positive & 2 & 2 & $>.05$ \\
& Negative & 6 & 6 & \\
Total & - & 56 & 56 & - \\
\hline
\end{tabular}

MP, moderate periodontitis; SP, slight periodontitis; SvP, severe periodontitis.

$P>.05$ indicates a nonstatistically significant difference. 
determinant of pulp sensibility. Pulp sensibility tests are noninvasive, standardized, reproducible, and low-cost techniques, making them ideal to assess pulp sensibility in daily clinical practice (23).

Pulp sensibility tests obtain a pulp response after the application of different stimuli, and they are the most indicated approaches for a differential diagnosis (5). Two tests to assess pulp sensibility were used in this study. Our results showed that thermal cold and electric tests showed no statistically significant differences. The small sample size should be considered a limitation of this study. Therefore, although it may have some limitations, this study is relevant, is clinically important, and provides useful scientific data. In addition, because of the scarcity of studies that assess the influence of periodontitis on the pulp, further studies are necessary to better clarify the complex relationship between the pulp and the periodontium. Specifically, such studies should consider the histopathology of the involved tissues. In conclusion, pulp clinical involvement with a negative response to thermal cold and electric testing was observed only in the most advanced stages of chronic periodontitis with apical involvement.

\section{Acknowledgments}

The authors deny any conflicts of interest related to this study.

\section{References}

1. Paul BF, Hutter JW. The endodontic-periodontal continuum revisited: new insights into etiology, diagnosis and treatment. J Am Dent Assoc 1997;128: 1541-8.

2. Meng HX. Periodontic-endodontic lesions. Ann Periodontol 1999;4:84-90.

3. Ingle JI, Bakland LK. Endodontics. Baltimore: Lea \& Feabinger; 1994:472-7.

4. Zuza EP, Carrareto AL, Lia RC, et al. Histopathological features of dental pulp in teeth with different levels of chronic periodontitis severity. ISRN Dent 2012;2012:271350.

5. Zehnder M, Gold SI, Hasselgren G. Pathologic interactions in pulpal and periodontal tissue. J Clin Periodontol 2002;29:663-71.
6. Jafarzadeh H, Abbott PV. Review of the pulp sensibility tests. Part I: general information and thermal tests. Int Endod J 2010;43:738-62.

7. Petersson K, Söderström C, Kiani-Anaraki M, Lévy G. Evaluation of the thermal and electrical tests to register pulp vitality. Endod Dent Traumatol 1999;15:127-31.

8. Weisleder R, Yamauchi S, Caplan DJ, et al. The validity of pulp testing: a clinical study. J Am Dent Assoc 2009;140:1013-7.

9. Peters DD, Baumgartner JC, Lorton L. Adult pulpal diagnosis. I. Evaluation of the positive and negative responses to cold and electrical pulp tests. J Endod 1994; 20:506-11.

10. Jafarzadeh H, Abbott PV. Review of pulp sensibility tests. Part II: electric pulp tests and test cavities. Int Endod J 2010;43:945-58.

11. American Academy of Periodontology. Consensus report: chronic periodontitis. Ann Periodontol 1999; 4:38.

12. Armitage GC. The complete periodontal examination. Periodontol 2000 2004;34: $22-33$.

13. Aass AM, Rossow I, Preus HR, Gjermo P. Incidence of early periodontitis in a group of young individuals during 8 years: associations with selected potential predictors. J Periodontol 1994;65:814-9.

14. Ramsay DS, Artun J, Martinen SS. Reliability of pulpal blood-flow measurement utilizing laser Doppler flowmetry. J Dent Res 1991;70:1427-30.

15. Hall CJ, Freer TJ. The effects of early orthodontic force application on pulp test responses. Aust Dent J 1998;43:359-61.

16. Zuza EP, Toledo BE, Hetem $S$, et al. Prevalence of different types of accessory canals in the furcation area of third molars. J Periodontol 2006;77:1755-61.

17. Zuza EP, Carrareto AL, Pontes AE, et al. Chronic periodontal disease may influence the pulp sensitivity response: clinical evaluation in consecutive patients. ISRN Dent 2012;2012:246875

18. Langeland K, Rodrigues H, Dowden W. Periodontal disease, bacteria, and pulpal histopathology. Oral Surg Oral Med Oral Pathol 1974;37:257-70.

19. Mazur B, Massler M. Influence of periodontal disease on the dental pulp. Oral Surg Oral Med Oral Pathol 1964;17:592-603.

20. Jansson L, Ehnevid H, Blomlöf L, et al. Endodontic pathogens in periodontal disease augmentation. J Clin Periodontol 1995;22:598-602.

21. Jansson L, Ehnevid H, Lindskog S, Blomlöf L. The influence of endodontic infection on progression of marginal bone loss in periodontitis. J Clin Periodontol 1995;22: 729-34.

22. Badillo F, Brouillet JL. Aging of the dental pulp. Rev Fr Endod 1991;10:41-54.

23. Chambers IG. The role and methods of pulp testing in oral diagnosis. Int Endod J 1982;15:1-15. 